University of Nebraska - Lincoln

DigitalCommons@University of Nebraska - Lincoln

\title{
Magnetically driven hyper-velocity launch capability at the Sandia Z accelerator
}

\author{
R. W. Lemke \\ Sandia National Laboratory, Albuquerque, NM \\ M. D. Knudson, \\ Sandia National Laboratory, Albuquerque, NM \\ J. P. Davis \\ Sandia National Laboratory, Albuquerque, NM
}

Follow this and additional works at: https://digitalcommons.unl.edu/usdoepub

Part of the Bioresource and Agricultural Engineering Commons

Lemke, R. W.; Knudson, M. D.; and Davis, J. P., "Magnetically driven hyper-velocity launch capability at the Sandia Z accelerator" (2011). US Department of Energy Publications. 121.

https://digitalcommons.unl.edu/usdoepub/121

This Article is brought to you for free and open access by the U.S. Department of Energy at DigitalCommons@University of Nebraska - Lincoln. It has been accepted for inclusion in US Department of Energy Publications by an authorized administrator of DigitalCommons@University of Nebraska - Lincoln. 


\title{
Magnetically driven hyper-velocity launch capability at the Sandia Z accelerator
}

\author{
R.W. Lemke*, M.D. Knudson, J-P Davis \\ Sandia National Laboratory, Albuquerque, NM 87185, USA
}

\section{A R T I C L E I N F O}

\section{Article history:}

Available online 10 November 2010

\section{Keywords:}

Stripline

Flyer Plate

Magnetic Launch

Quasi-isentropic

Hugoniot

\begin{abstract}
A B S T R A C T
The intense magnetic field generated by the $\mathrm{Z}$ accelerator at Sandia National Laboratories is used as a pressure source for material science studies. A current of $\sim 20$ MA can be delivered to the loads used in experiments on a time scale of $\sim 100-600$ ns. Magnetic fields (pressures) exceeding $1200 \mathrm{~T}$ (600 GPa) have been produced in planar configurations. In one application we have developed, the magnetic pressure launches a flyer plate to ultra-high velocity in a plate impact experiment; equation of state data is obtained on the Hugoniot of a material that is shock compressed to multi-megabar pressure. This capability has been enhanced by the recent development of a planar stripline configuration that increases the magnetic pressure for a given current. Furthermore, the cross sectional area of a stripline flyer plate is larger than in previous coaxial loads; this improves the planarity of the flyer thereby reducing measurement uncertainty. Results of experiments and multi-dimensional magneto hydrodynamic (MHD) simulation are presented for ultra-high velocity aluminum and copper flyer plates. Aluminum flyer plates with dimensions $\sim 25 \mathrm{~mm}$ by $\sim 13 \mathrm{~mm}$ by $\sim 1 \mathrm{~mm}$ have been launched to velocities up to $\sim 45 \mathrm{~km} / \mathrm{s}$; for copper the peak velocity is $\sim 22 \mathrm{~km} / \mathrm{s}$. The significance of these results is that part of the flyer material remains solid at impact with the target; an accomplishment that is made possible by shaping the dynamic pressure (current) ramp so that the flyer compresses quasi-isentropically (i.e., shocklessly) during acceleration.
\end{abstract}

Published by Elsevier Ltd.

\section{Introduction}

The intense magnetic field generated by the $\mathrm{Z}$ accelerator [1] at Sandia National Laboratories is used as a pressure source for material science studies [2-11]. A current of 20 MA can be delivered to the short circuited loads used in experiments on a time scale of $\sim 100-600 \mathrm{~ns}$; this spread in time to peak current is enabled by the ability to shape the current wave form via triggering 18 independent pairs of gas switches [1,3,6]. Magnetic fields (pressures) exceeding $1200 \mathrm{~T}$ (600 GPa) have been produced in planar configurations.

In one application we have developed, the magnetic pressure launches a flyer plate to ultra-high velocity in a plate impact experiment; equation of state (EOS) data is obtained on the Hugoniot of a material that is shock compressed to multi-megabar pressure [4,5,8-11]. In these experiments a velocity interferometry (VISAR) technique is used to measure the flyer velocity [8], and the velocity of the shock produced in the target material. These velocity

\footnotetext{
* Corresponding author. Tel.: +1 505 8457423; fax: +1 5058448467

E-mail address: rwlemke@sandia.gov (R.W. Lemke).
}

measurements are used in the shock jump conditions under the assumption that the material state of the flyer at impact is known in order to calculate the pressure and density of the shocked material [8]. Also, measurement uncertainty [8] is improved if the flyer is ballistic at impact. Thus, it is desirable to accelerate the flyer to peak velocity in a manner that preserves some material (on the free surface [target] side) in a solid state at impact; a non trivial accomplishment at multi-megabar pressure.

Shock formation in the flyer during acceleration and Joule heating associated with the magnetic field are two mechanisms that can melt the entire flyer before it impacts the target. As the accelerating magnetic field diffuses into the flyer (toward the free surface), the associated Joule heating melts and vaporizes the flyer material. Thus, the entire flyer burns up if the flight time to target is longer than the flyer thickness divided by the magnetic diffusion rate. We have previously verified that the magnetic diffusion front is slower than the stress wave launched into the flyer [11]; were it otherwise the magnetic launch technique would not be feasible.

Shock formation in the flyer during acceleration can melt or vaporize the entire flyer, depending on magnitude; aluminum melts at a shock pressure of $\sim 1.2$ Mbar. To preclude shock formation during acceleration, the current rise time to peak current is shaped 

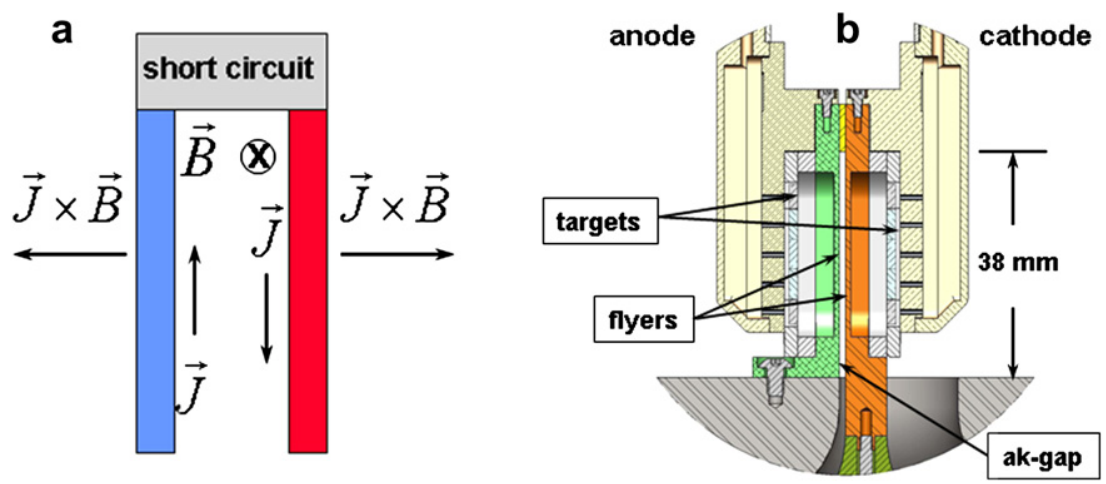

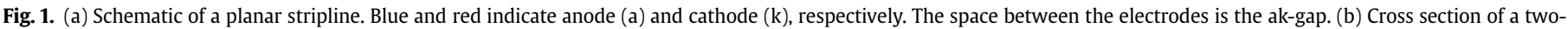

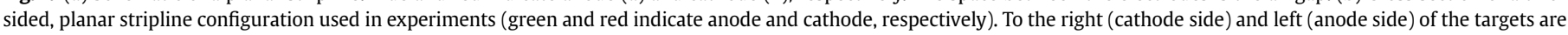
the inserts and shielding for the VISAR optical fibers. Electrical power enters the stripline at the bottom.

so that the flyer material compresses quasi-isentropically (shocklessly) during its flight to the target [9]. Shaping the current pulse to preclude shock formation during acceleration, and the ability to design the required pulse shape and flight distances using ALEGRA [12] magneto hydrodynamics (MHD) simulations are the key factors that enable us to magnetically accelerate flyers to ultra-high velocity using multi-megabar pressures while preserving part of the flyer in a state required for accurate EOS measurements.

This capability has been enhanced significantly by the refurbishment of the Z accelerator [1,3], and by the recent development of a planar stripline configuration. The refurbishment increased the current that can be delivered to a short circuited load by $\sim 25 \%$, and improved the pulse shaping capability. The stripline increases the magnetic pressure in the load for a given current, and allows for larger area flyers (than previous coaxial loads); this improves their planarity thereby reducing measurement uncertainty. To date, a $900 \mu \mathrm{m}$ thick, aluminum (Al) stripline flyer plate with cross sectional dimensions $\sim 25 \mathrm{~mm}$ by $\sim 13 \mathrm{~mm}$ was shocklessly accelerated to a record peak velocity of $45 \mathrm{~km} / \mathrm{s}$ in $\sim 300 \mathrm{~ns}$ using a shaped current that produced a peak magnetic pressure (field) of $6.3 \mathrm{Mbar}$ ( $1263 \mathrm{~T}$ ); this velocity is a $30 \%$ increase over the maximum that was produced using a coaxial load on $\mathrm{Z}$ before the refurbishment [9].

In the following sections details pertaining to the planar stripline configuration used in ultra-high velocity flyer plate impact experiments on $\mathrm{Z}$ are presented; included are VISAR velocity measurements from state-of-the-art experiments with $\mathrm{Al}$ and copper $(\mathrm{Cu})$ flyers, results from corresponding MHD simulations, and the method used to define the shape for the ramped current drive. Also, results from MHD simulations are used to elucidate phenomena that ultimately limit the peak magnetic pressure that can be produced in a planar load.

\section{The stripline flyer plate load}

The basic stripline load is shown schematically in Fig. 1(a); it is composed of two rectangular slabs (usually aluminum) of height $\mathrm{H}$ (vertical direction), width $\mathrm{W}$ (perpendicular to plane of figure), and thickness $\Delta$, which comprise the electrodes (anode and cathode) that carry current into and through the load. Typical stripline dimensions are $\mathrm{H} \sim 38 \mathrm{~mm}, \mathrm{~W} \sim 11-15 \mathrm{~mm}$, and $\Delta=900 \mu \mathrm{m}$. The electrodes are connected at one end (the top) by another conductor; this short circuit configuration maximizes the magnetic field in the ak- (anode-cathode) gap $(g \sim 1 \mathrm{~mm})$. The electrodes are the flyer plates. Also shown in Fig. 1(a) are the directions of current density $(J)$, magnetic field $(B)$, and the Lorentz force $(J x B)$ that accelerates the flyer plates. The stripline is open at the termini perpendicular to the plane of the page. Consequently the magnetic field is not confined to the ak-gap; it wraps around each electrode similar to a current carrying wire, and current flows on the external surfaces of the electrodes. The magnetic field amplitude decreases rapidly with increasing distance outside the ak-gap.

For small electrode displacements and $\mathrm{g}<<\mathrm{W}$ the magnetic field in the ak-gap is given by

$B=f \mu_{0} I /(g+W)$

where $I$ is the time dependent input current, $\mu_{0}$ the permeability of free space, and $f$ is a factor that accounts for current flowing outside the ak-gap $(f \leq 1)$. Multi-megabar magnetic pressures produce significant electrode motion; consequently, in actuality $g, W$, and $f$ are functions of time. The magnetic pressure on the electrodes (flyers) is $P_{B}=B^{2} / 2 \mu_{0}$. The ak-gap increases as the flyers are pushed outward, which decreases the magnetic pressure on the flyer drive surface for a given current.
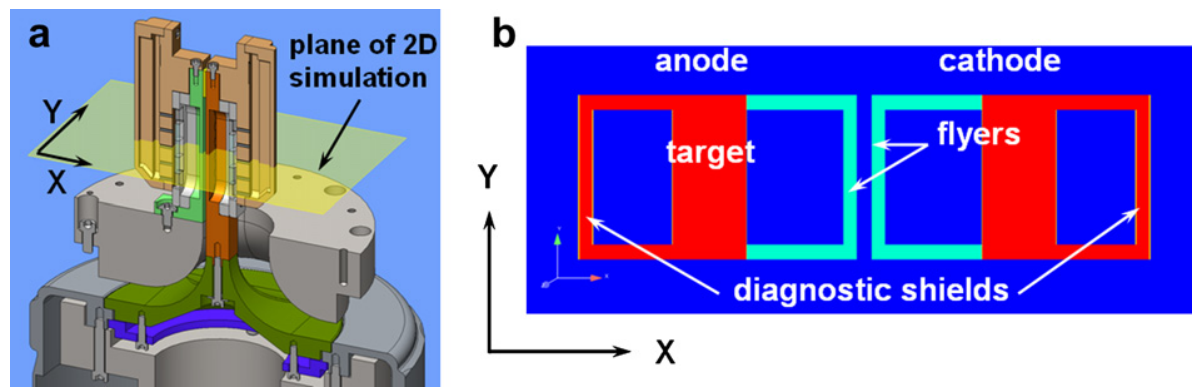

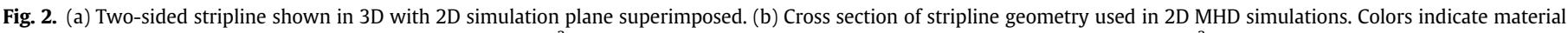
density which varies from 0 for void (dark blue) to $8930 \mathrm{~kg} / \mathrm{m}^{3}$ for copper (red); the flyer material is aluminum $\left(\right.$ density $=2700 \mathrm{~kg} / \mathrm{m}^{3}$ ). 

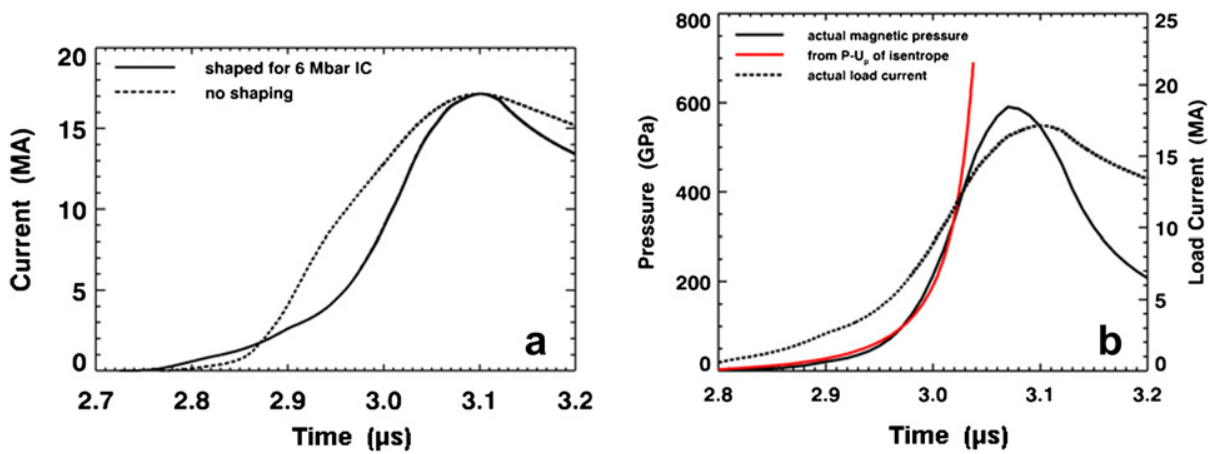

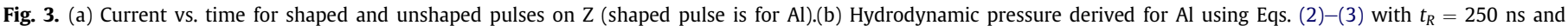
$P_{\max }=7 \mathrm{Mbar}$ (red curve), and magnetic pressure (black solid line) produced by a feasible shaped current (dashed line).

Fig. 1(b) is a schematic of a two-sided planar stripline load used in flyer plate impact experiments. In this configuration both electrodes are flyer plates, and there are two sets of five targets opposite each flyer plate. A VISAR measurement of flyer velocity and shock velocity (in a target) is made at each target location; hence, in this configuration ten simultaneous plate impact experiments are possible in one shot of the accelerator. Because current flows on the outside surfaces of the stripline it is necessary to shield the VISAR optical fibers in what is essentially a Faraday cage; furthermore, they must be shielded from the bremsstrahlung produced by electrons flowing in the power feed below the entrance to the stripline.

A one-sided version of the stripline is also used in experiments. In this configuration the cathode side flyer (electrode) and targets are replaced by a single, thick $(\sim 1 \mathrm{~cm})$ block of tungsten; hence, there is only one flyer and one set of targets (on the anode side). With this change the ak-gap increases more slowly during the current pulse, which results in higher magnetic pressure than in a two-sided stripline for the identical current pulse. A one-sided stripline was used to produce the highest velocity aluminum flyer to-date $(45 \mathrm{~km} / \mathrm{s})$.

\subsection{Computational model of the stripline for MHD simulation}

Stripline flyer plate experiments are designed and analyzed using a predictive MHD modeling capability we have developed for material science studies on the $Z$ accelerator [9-12]. The crux of this capability is the multi-dimensional, resistive MHD code ALEGRA [12]; the DAKOTA optimization code [13] and BERTHA transmission line code [14] are also key elements. The ALEGRA MHD simulation model, which incorporates accurate models of material properties and power flow in Z, was developed and validated using data from experiments with a coaxial flyer plate configuration [9-11]. Details pertaining to the computational modeling can be found in the references.
The stripline configuration used in 2D MHD simulations is shown schematically in Fig. 2. Fig. 2(a) shows the simulation plane superimposed on a 3D schematic of a two-sided stripline; Fig. 2(b) shows a cross section of the stripline in this plane that represents the geometry in 2D simulations. We have verified by experiment and 3D electromagnetic (EM) simulation that the magnetic field in the stripline increases with height [15]; in the one-sided version of Fig. 2(a) it varies by $\sim 2.2 \%$ over the distance spanned by the five targets (corresponding magnetic pressure varies by $\sim 5 \%$ ). Three dimensional EM simulations show that the 3D and 2D current (magnetic field) distributions are the same at the height of the middle target sample; thus, 2D MHD simulations are an accurate representation of the experiment for measurements taken at the mid-height location.

In Fig. 2(b) the stripline width in the $y$-direction is $W=11 \mathrm{~mm}$; flyer thicknesses are $\Delta=900 \mu \mathrm{m}$; ak-gap, $g=1 \mathrm{~mm}$; flight distance to target is $6 \mathrm{~mm}$ on both sides. The direction of current flow is perpendicular to the plane of the figure ( $z$-direction). Since there is significant conductor motion and deformation during the current pulse (as will be shown below), a self-consistent simulation model must be at least $2 \mathrm{D}$ and include a circuit representation of power flow in Z [10].

\subsection{Shaping the current pulse for shockless acceleration of stripline flyer plates}

For multi-megabar magnetic pressures the rise time of the current pulse is shaped in order to preclude shock formation in the flyer during acceleration. The ramp to peak current is shaped so that the flyer compresses quasi-isentropically during acceleration. A time dependent pressure ramp is derived using the compression isentrope for the relevant flyer material; then 2D MHD simulations of the stripline are performed to obtain a current that produces the desired pressure ramp. Finally, the BERTHA circuit model of Z [3] is

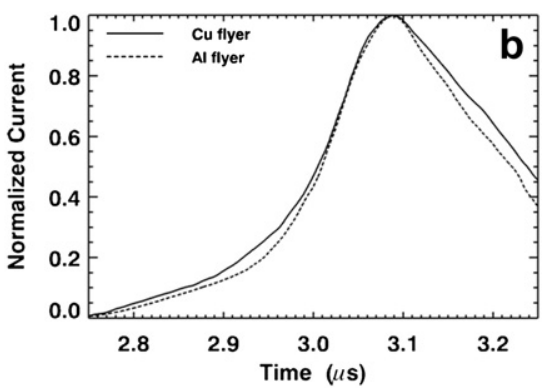

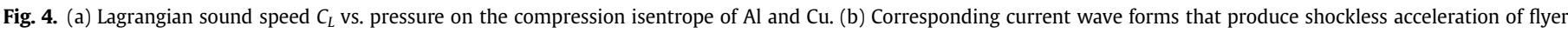
plates for a peak magnetic drive pressure of $\sim 6$ Mbar. 

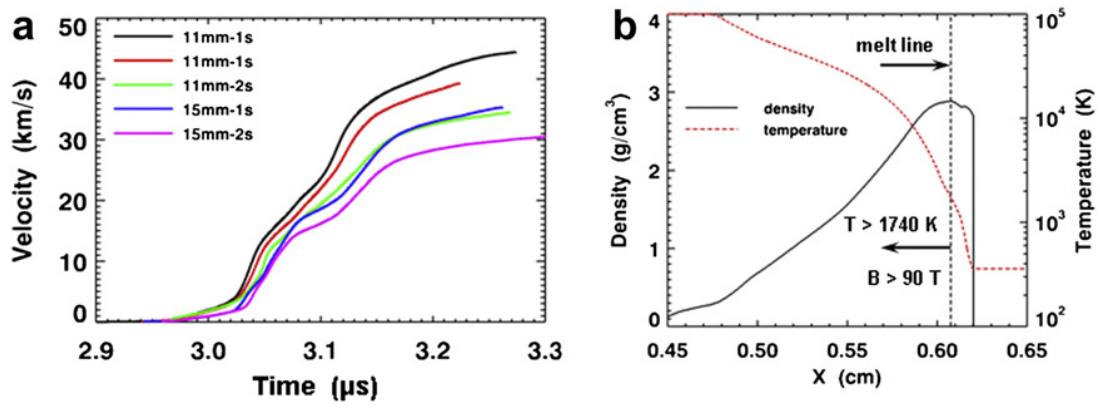

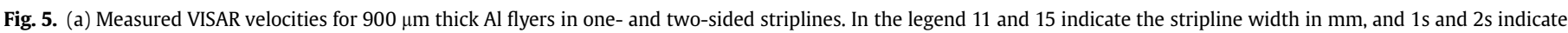
a one- or two-sided configuration. (b) Simulated density and temperature in the $11 \mathrm{~mm}-1 \mathrm{~s}, 40 \mathrm{~km} / \mathrm{s}$ aluminum flyer just before impact with a target.

run using the time dependent current and inductance from MHD simulations as input in order to verify that the current is feasible, and to determine the machine timing that produces the current.

Given the pressure $(P)$, density $(\rho)$, and sound speed $\left(C_{S}\right)$ on the isentrope of a given material, and a desired rise time $\left(t_{R}\right)$ to a peak pressure $\left(P_{\max }\right)$, the shock up distance $\left(X_{S}\right)$ in the Lagrangian frame of the flyer is given by

$X_{S}=t_{R} C_{L N} C_{L O} /\left(C_{L N}-C_{L O}\right)$

where $C_{L}(P)=C_{S}(P) \rho(P) / \rho_{0}$ is the pressure dependent Lagrangian sound speed; the subscripts $\mathrm{N}$ and 0 indicate initial and final pressures (times) on the isentrope (pressure ramp), which has been divided into $\mathrm{N}$ intervals. The pressure $P_{n}$ at time $t_{n}$ on the ramp is obtained using

$t_{n}=t_{R}-X_{S}\left[C_{L N}\left(P_{\max }\right)-C_{L n}\left(P_{n}\right)\right] / C_{L N}\left(P_{\max }\right) C_{L n}\left(P_{n}\right)$

Given this pressure ramp, a flyer with thickness $\Delta<X_{S}$ will not shock up during acceleration for magnetic pressures $P_{B} \leq P_{\max }$. Alegra 2D MHD simulations are used to convert the pressure ramp into a feasible current [9], and determine the flight distance needed to establish the required flyer conditions before impact with a target. Shaped and unshaped current and pressure drives used in stripline experiments with $900 \mu \mathrm{m}$ thick Al flyers are plotted in Fig. 3. The peak accelerating magnetic pressure is $\sim 600 \mathrm{GPa}$. The currents in Fig. 3 are measurements; the magnetic pressure is from a $2 \mathrm{D}$ simulation.

Results for a $200 \mu \mathrm{m}$ thick $\mathrm{Cu}$ flyer are shown in Fig. 4. The Lagrangian sound speeds $C_{L}(P)$ on the compression isentropes of $\mathrm{Al}$ and $\mathrm{Cu}$ are plotted in Fig. 4(a); the corresponding shaped current wave forms (valid for $P_{\max }=7$ Mbar) are plotted in Fig. 4(b). Comparison of these figures reveals that the rate at which the sound speed increases with increasing pressure on the isentrope determines the rate at which the applied current can change. Departures from these shapes by a few percent can result in shock formation, especially for times $2.86 \leq t \leq 3.0 \mu \mathrm{s}$.

\subsection{Experimental and computational results}

Measured VISAR velocities are plotted in Fig. 5(a) for $900 \mu \mathrm{m} \mathrm{Al}$ flyers in $11 \mathrm{~mm}$ and $15 \mathrm{~mm}$ wide, one- and two-sided striplines with $1 \mathrm{~mm}$ ak-gaps. Quartz and/or sapphire targets were present in all shots, located approximately $6 \mathrm{~mm}$ from the flyer free surface. The highest velocity (black curve) was achieved by repeating a shot of the one-sided, $11 \mathrm{~mm}$ wide stripline at slightly larger capacitor charge voltage, which increases the current. It is clear from the velocities that pulse shaping has precluded shock formation. In all cases post-shot analysis using MHD simulation shows that a fraction of the aluminum flyer is still solid at impact with the target. The simulated density and temperature versus position in the $\sim 40 \mathrm{~km} / \mathrm{s}$ Al flyer [red curve in Fig. 5(a)] just before impact is plotted in Fig. 5(b). The Al EOS is used to establish the melt line [9]; consistent with previous results, the location of the melt line is well correlated with where the magnetic field has reached an amplitude of $90 \mathrm{~T}$ [9]. Approximately $124 \mu \mathrm{m}$ of $\mathrm{Al}$ is still solid at impact. This flyer produced measured shock pressures of 15.7 and 20.7 Mbar in $\alpha$-quartz [4] and sapphire targets, respectively. The time dependent shock measurement in sapphire validated the simulated density profile.

Results from ALEGRA 2D MHD simulations of the $11 \mathrm{~mm}$ wide, two-sided stripline shot [11 mm-2s case in Fig. 5(a)] are presented in Figs. 6 and 7. Current, VISAR velocity, and simulated velocity versus time are plotted in Fig. 6(a); the solid green dots and black asterisks are reference points for the results in Fig. 7. The current is determined using the VISAR velocity in conjunction with 2D and 1D MHD simulations, and DAKOTA optimization [9]. First, a DAKOTA
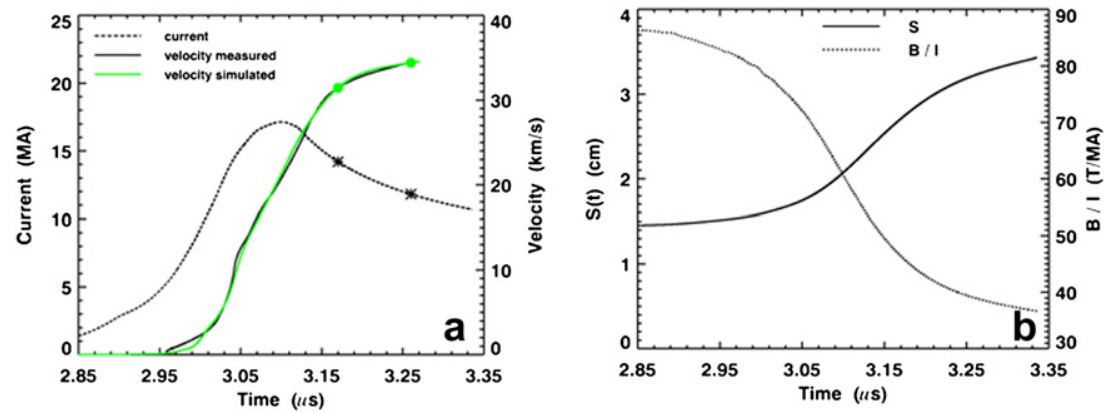

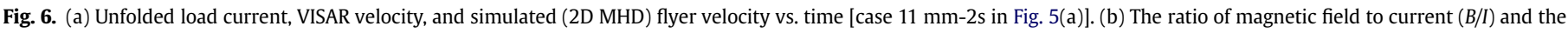
geometric factor $S$ vs. time. 

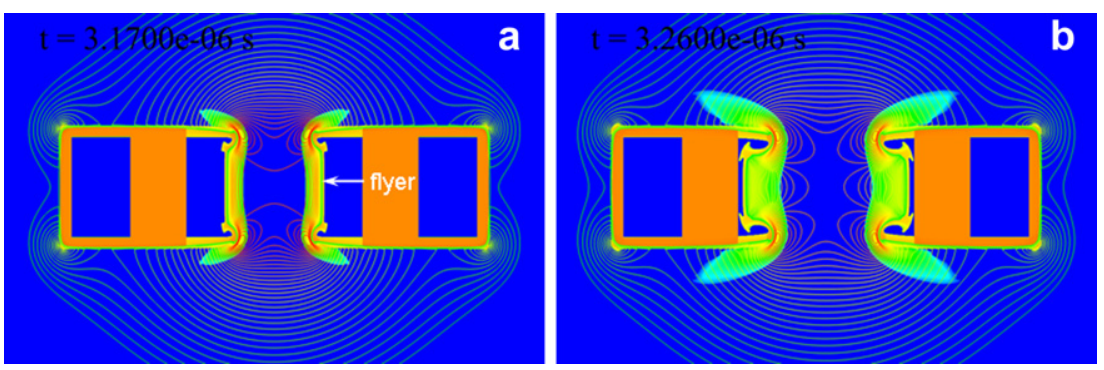

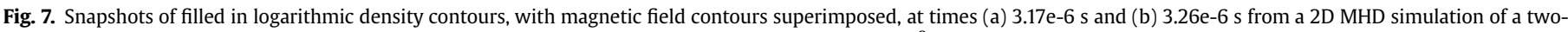

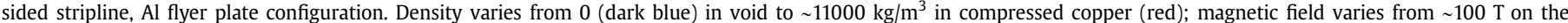
perimeter of the figures to $\sim 1000 \mathrm{~T}$ at the edges of the ak-gap.

optimization problem is performed using ALEGRA high resolution ( $\sim \mu \mathrm{m}$ spatial cells), 1D Lagrangian, MHD simulations in conjunction with a measured VISAR velocity. This step produces the precise magnetic pressure drive that accelerates the flyer, and a simulated flyer velocity that is within the measurement uncertainty $(\sim 1 \%)$; the state of the flyer material at any time during the current pulse is deduced from this calculation [as exemplified in Fig. 5(b)]. Second, the magnetic field time history from the first step is used in conjunction with a series of 2D MHD simulations to obtain the load current [9]. This unfolded load current, which is not measured directly in experiments on $Z$, is an accurate representation of the current that actually reaches the stripline [based on agreement between simulated (2D) and measured flyer velocities, in Fig. 6(a) for example].

Fig. 6(b) includes time histories of the geometric factor $S(t)=[g(t)+W(t)] / f$ (see Eq. (1)), and $B / I=\mu_{0} / S$ in the ak-gap, where $I$ is the current in Fig. 6(a) and $B$ is evaluated at the mid-point of the ak-gap [see Fig. 2(b)]. This plot indicates that conductor motion produces a reduction in magnetic field (pressure) for a given current. Consequently, as current is increased on $\mathrm{Z}$ to achieve higher pressure and flyer velocity it is observed that the scaling of peak velocity with current changes continuously from quadratic to linear.

In Fig. 7 snapshots of the stripline density (i.e., filled in, logarithmic density contours) with contours of magnetic field superimposed are shown for two times (3.17e-6 s and 3.26e-6s) in the 2D MHD simulation [see reference points in Fig. 2(a)]. Refer to Fig. 2(b) for the initial state of the stripline, and locations of flyers and targets. In Fig. 7(a) the location of the flyer on the cathode side is indicated by an arrow. Fig. 7(b) shows the state of the configuration when the flyers have reached peak velocity, just before impact with the target. The density and temperature profiles in the flyer are similar to those plotted in Fig. 5(b); $\sim 120 \mu \mathrm{m}$ of aluminum is still solid at impact. According to the 2D MHD simulations the flyer free surface is planar to within $0.5 \%$ over a distance of $4.8 \mathrm{~mm}$, which is a significant improvement compared to coaxial load flyers [10].
Enhancement of the current density at corners results in jetting of aluminum plasma, particularly at the top and bottom corners in the ak-gap region. At impact the state of aluminum varies from a relatively cool solid at $\sim 500 \mathrm{~K}$ near the flyer free surface to low density $\left(\sim 0.1-1.0 \mathrm{~kg} / \mathrm{m}^{3}\right)$ plasma at $\sim 100,000 \mathrm{~K}$ in the ablation tail on the drive side of the flyer, and in the jets external to the ak-gap. If this jetting is present in experiments it has negligible effect on the VISAR diagnostics, which are completely enclosed by metal shielding [Fig. 1(b),2, and 7].

It is also clear in Fig. 7 that expansion of the ak-gap $(g)$ is the predominant geometric effect that produces the monotonic decrease (increase) in $B / I$ (the geometric factor $S$ ) in Fig. 6(b). However, the plasma jetting at the edges of the electrodes decreases (increases) the fraction of the total load current flowing inside (outside) the ak-gap. This effect contributes to the decrease (increase) in $B / I(S)$ shown in Fig. 6(b).

\subsubsection{Results for copper flyers}

We are developing striplines composed of copper flyers in order to increase the dwell time in plate impact experiments by taking advantage of the slower wave speed compared to $\mathrm{Al}$; for example, the speed of a $\sim 29.2$ Mbar shock in $\mathrm{Cu}$ is reduced by a factor of $\sim 0.59$ compared to the speed in $\mathrm{Al}(40.9 \mathrm{~km} / \mathrm{s})$. The flyer plate is actually a composite of $\mathrm{Al}$ and $\mathrm{Cu}$ in order to keep the magnetic field from diffusing into the $\mathrm{Cu}$ as long as possible. Copper is electroplated onto a base plate of $\mathrm{Al}$ and then machined to the desired dimension. We have performed several shots of striplines that included two flyers, each composed of a $200 \mu \mathrm{m}$ thick Cu layer on a base plate of $\mathrm{Al}$, $800 \mu \mathrm{m}$ thick. This experiment was designed using 2D simulation, and used the shaped wave form for $\mathrm{Cu}$ in Fig. 4(b); measured and simulated velocities are plotted in Fig. 8(a). There is clearly no shock formation despite the $4.3 \mathrm{Mbar}$ peak accelerating magnetic pressure. $\mathrm{An} \mathrm{Al} / \mathrm{Cu}$ flyer produces a larger shock in a Cu target (for example) than a pure Al flyer with the same speed, which is due to the higher density of $\mathrm{Cu}$. This is illustrated graphically in Fig. 8(b). Fig. 8(b) is a plot of pressure versus particle (mass) velocity on the Hugoniots of
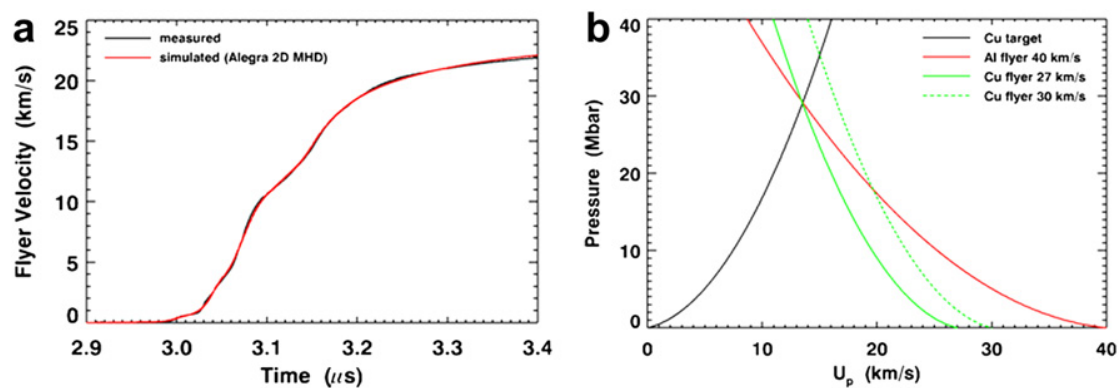

Fig. 8. (a) Measured and simulated velocities for an $800 / 200 \mu \mathrm{m} \mathrm{Al} / \mathrm{Cu}$ stripline flyer plate. (b) Pressure vs. particle (mass) velocity on the Hugoniots of Cu and $\mathrm{Al}$. 
$\mathrm{Al}$ and $\mathrm{Cu}$. A $27 \mathrm{~km} / \mathrm{s} \mathrm{Cu}$ flyer produces the same pressure in a $\mathrm{Cu}$ target as does a $40 \mathrm{~km} / \mathrm{s}$ Al flyer. Calculations indicate that an $\mathrm{Al} / \mathrm{Cu}$ flyer velocity exceeding $30 \mathrm{~km} / \mathrm{s}$ is feasible on $\mathrm{Z}$.

\section{Conclusions}

We have developed a new stripline flyer plate configuration for experiments on $Z$ that produced a record $45 \mathrm{~km} / \mathrm{s}$ aluminum flyer in a state that can be used for accurate EOS measurements. The development of striplines that include composite $\mathrm{Al} / \mathrm{Cu}$ flyer plates is ongoing; experiments have already produced a $22 \mathrm{~km} / \mathrm{s}, 800 /$ $200 \mu \mathrm{m} \mathrm{Al} / \mathrm{Cu}$ flyer plate; $30 \mathrm{~km} / \mathrm{s}$ or greater is feasible. In plate impact experiments on $\mathrm{Z}$ it is now possible to produce shock pressures $\sim 30-40$ Mbar in targets of interest. These results are possible because of our ability to accurately design and produce shaped current pulses on the $\mathrm{Z}$ accelerator.

\section{Acknowledgements}

We thank Dustin Heinz Romero for producing schematics of the stripline flyer plate configuration, and the large group of people who make experiments possible on Z. Sandia is a multi-program laboratory operated by Sandia Corporation, a Lockheed Martin Company, for the United States Dept. of Energy under Contract DEAC04-94AL85000.

\section{References}

[1] McDaniel DH, Mazarakis MG, Bliss DE, et al. The ZR refurbishment project. Proc. 5th Int. Conf. on Dense Z-Pinches, Davis C, Deeney, Pereira NR (AIP, Melville, NY, 2002), editors, Albuquerque, NM, June 23-28, 2002: p. 23.
[2] Matzen KM, Sweeney MA, Adams RG, et al. Pulsed-power-driven high energy physics and inertial confinement fusion research. Phys Plasmas 2005;12: 055503.

[3] Struve KW, Bennet LF, Chavez TF, et al. Circuit-code modeling of the refurbished $\mathrm{Z}$ accelerator: comparison of measurements with predictions. Proc. 2008 IEEE International Power Modulators and High Voltage Conference. Kirkici H (IEEE, New York, NY), editor, Las Vegas, NV, 2008: p. 94.

[4] Knudson MD, Desjarlais MP. Shock compression of quartz to $1.6 \mathrm{TPa}$ : redefining a pressure standard. Phys Rev Lett 2009;103:225501.

[5] Knudson MD, Desjarlais MP, Dolan DH. Shock-wave exploration of the highpressure phases of carbon. Science 2008;322:1822-5.

[6] Davis JP, Knudson MD. Multi-megabar measurement of the principal quasiisentrope for tantalum. Shock Compression of Condensed Matter - 2009. Elert ML, Buttler WT, Furnish MD, Anderson WW, Proud WG, editors. (AIP Conf. Proc. 1195, Melville, NY, 2009), Nashville, TN, June 28 - July 3, 2009: p. 673-676.

[7] Davis JP, Deeney C, Knudson MD, Lemke RW, Pointon TD, Bliss DE. Magnetically driven isentropic compression to multimegabar pressures using shaped current pulses on the Z accelerator. Phys Plasmas 2005;12:056310.

[8] Knudson MD, Lemke RW, Hayes DB, Hall CA, Deeney C, Asay JR. Near-absolute Hugoniot measurements in aluminum to $500 \mathrm{GPa}$ using a magnetically accelerated flyer plate technique. J Appl Phys 2003;94(7):4420-31.

[9] Lemke RW, Knudson MD, Bliss DE, Cochrane K, Davis JP, Giunta AA, et al. Magnetically accelerated, ultrahigh velocity flyer plates for shock wave experiments. J Appl Phys 2005;98:073530.

[10] Lemke RW, Knudson MD, Robinson AC, Haill TA, Struve KW, Asay JR, et al. Self-consistent, two-dimensional, magnetohydrodynamics simulations of magnetically driven flyer plates. Phys Plasmas 2003;10(5):1867-74.

[11] Lemke RW, Knudson MD, Hall CA, Haill TA, Desjarlais MP, Asay JR, et al. Characterization of magnetically accelerated flyer plates. Phys Plasmas 2003;10(4):1092-9.

[12] Robinson AC, Brunner TA, Carrol S, et al. ALEGRA: an arbitrary LagrangianEulerian multimaterial, multiphysics code. 46th AIAA Aerospace Sciences Meeting and Exhibit, Reno, NV; 2008. AAIAA 2008-12351-39.

[13] Eldred MS, Giunta AA, van Bloeman Waanders BG. Structural and multidisciplinary optimization, vol. 27. Berlin: Springer-Verlag; 2004. 97.

[14] Hinshelwood DD. BERTHA- A versatile transmission line and circuit code. Naval Research Laboratory Memorandum; 1983. Report No. 5185.

[15] Seidel DB, Langston WL, Coats RS, Knudson MD, Lemke RW, Davis JP. An optimization study of stripline loads for isentropic compression experiments. Proceedings of the 2009 IEEE Pulsed Power Conference. Washington, D. C., June 2009, ISBN 978-1-4244-4065-8. 